\title{
Numerical Modeling of the Internal Dispersive Shock Wave in the Ocean
}

\author{
Tatiana Talipova, ${ }^{1,2}$ Efim Pelinovsky, ${ }^{1,2}$ Oxana Kurkina, ${ }^{2}$ and Andrey Kurkin ${ }^{2}$ \\ ${ }^{1}$ Institute of Applied Physics, Russian Academy of Sciences, Nizhny Novgorod 603950, Russia \\ ${ }^{2}$ Department of Applied Mathematics, Nizhny Novgorod State Technical University n.a. R.E. Alekseev, \\ Nizhny Novgorod 603950, Russia \\ Correspondence should be addressed to Efim Pelinovsky; pelinovsky@gmail.com
}

Received 12 April 2015; Accepted 3 June 2015

Academic Editor: Chao Tao

Copyright (C) 2015 Tatiana Talipova et al. This is an open access article distributed under the Creative Commons Attribution License, which permits unrestricted use, distribution, and reproduction in any medium, provided the original work is properly cited.

\begin{abstract}
Numerical modeling of dispersive shock waves called solibore in a stratified fluid is conducted. The theoretical model is based on extended version of the Korteweg-de Vries equation which takes into account the effects of cubic nonlinearity and Earth rotation. This model is now very popular in the physical oceanography. Initial conditions for simulations correspond to the real observed internal waves of shock-like shape in the Pechora Sea, the Arctic. It is shown that a sharp drop (like kink in the soliton theory) in the depth of the thermocline is conserved at a distance of one-three kilometers, and then it is transformed into dispersive shock waves (shock wave with undulations).
\end{abstract}

\section{Introduction}

The theory of shock waves in fluid and gas in the framework of hyperbolic set of equations is rather well developed [1-3]. An effective method to analyze shock waves is the use of conservation laws for appropriate quantities on the front of the wave. The theory of shock waves in weakly dispersive media is more difficult, because the shock profile contains damped oscillations; it is called dispersive shock wave [4]. An example of a system that enables existence of dispersive shock wave is a well-known Korteweg-de Vries (KdV) equation with a small parameter at the term with higher derivative:

$$
u_{\tau}+u_{\xi}+\frac{1}{2}\left(u^{2}\right)_{\xi}=\mu u_{\xi \xi \xi}
$$

where $u(\xi, \tau)$ is a wave function, $\xi$ is spatial variable, $\tau$ is time, $\mu$ is a small parameter, and indices denote partial derivatives. All the quantities in (1) are assumed to be nondimensional. Here it is also possible to use the conservation laws; in particular for the Korteweg-de Vries equation there are an infinite number of conservation laws [5]. An effective method to analyze oscillating shock waves was proposed in the pioneering paper [6], and then it was developed in the series of studies by El and Kamchatnov with their coauthors [7-9]. The consideration of a weak dissipation (less than dispersion) results in a stabilization of dispersive shock wave, which then can propagate as travelling wave [10].

Internal waves in the ocean can serve as an excellent example of dispersive shock waves; such waves are called solibores [11-14]. Solibores are generated by baroclinic tides and were observed in many regions of the world ocean, especially in shallow water. Their modelling is usually performed in the framework of the Korteweg-de Vries-type equations (see the papers cited below). Here we will give the results of the modelling of internal solibore observed in Arctic waters. The special feature of Arctic regions from the point of view of the theory of nonlinear waves is the need to take into account rotation of Earth that leads to the increase of the order of evolutionary equations. In particular, the Korteweg-de Vries equation should be replaced by the Gardner-Ostrovsky equation [15], which is not integrable. In Section 2 we describe the observations of internal bore in the Pechora Sea (Arctic basin). The model of internal wave transformation, based on the Gardner-Ostrovsky equation, is given in Section 3. Coefficients of this equation are calculated for the two-layer model adapted for the real observed density stratification of 
the region of measurements; they are described in Section 4. Section 5 contains the results of numerical modeling of internal solibore. The main conclusions are given in Section 6.

\section{Observations of Internal Bore in the Pechora Sea (Arctic Basin)}

Internal bore was observed on the Barents Sea shelf in the socalled Pechora Sea. The bathymetric map is shown in Figure 1. The sea waters in this area are quite shallow; the depths are in the range of $10-30 \mathrm{~m}$.

Measurements of internal waves in the Pechora Sea were carried out during the 13th cruise of the R/V "Akademik Sergey Vavilov" in August 1998 [16]. The place of observations in the southern shallow water region of the Pechora Sea (depth $16 \mathrm{~m}$ ) is marked by red asterisk in Figure 1. Here we reproduce the description of the observed internal shock-like wave from [16]: "An abrupt upward shift of the thermocline for approximately $3 \mathrm{~m}$ was registered (front duration was about 2 minutes), indicating the passage of internal bore. Immediately after the bore forefront passage, the "yo-yo" profiles yielded intense temperature inversions with an amplitude up to $1^{\circ} \mathrm{C}$. Shortly thereafter the inversions disappeared, and the thermocline became completely smooth within 2 hours after the bore passage." We will not reproduce original record due to low quality, but its character will be visible then in Figure 4 .

In the present study we used averaged hydrographic data from climatological atlas [17], which was created exactly for the Arctic region. The nearest points to the point of records in this atlas were two points (numbered 3445 and 3493) with depths 15 and $25 \mathrm{~m}$. For these points temperature and salinity are given for 5 to 6 levels in vertical. On a base of these data the buoyancy (Brunt-Väisälä) frequency $N(z)$ was estimated:

$$
N(z)=\sqrt{-\frac{g d \rho_{0}}{\rho_{0} d z}},
$$

where $z$ is the vertical coordinate, $g$ is the gravity acceleration, and $\rho_{0}(z)$ is the undisturbed density of sea water. The profiles of $N(z)$ are shown in Figure 2.

\section{Theoretical Model of Internal Wave Transformation in a Rotating Ocean}

One of the main mechanisms of generation of short-period internal waves is the transformation of internal tides [12]. Therefore, a proper theoretical model should be able to describe this process of transformation of long waves into a train of short waves. An important point here is that Earth's rotation can notably affect these initially long waves. This impact apparently is particularly large at higher latitudes and in the case of wave propagation over long distances. The effect of background rotation generally causes a short internal wave to decay through the radiation of inertia-gravity waves; see the review $[18]$ and the recent studies $[17,19]$. In practice, the time scale for this decay is one or two inertial periods.

Several studies $[16,20,21]$ address theoretical models that include rotational effects on the same order (of weakness)

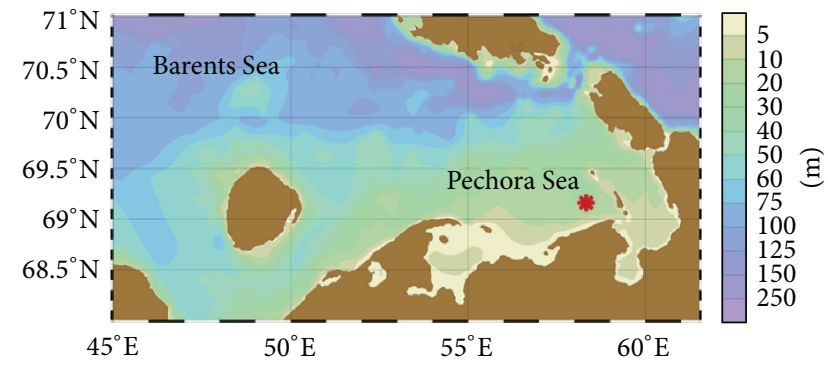

FIgure 1: Bathymetric map of the Pechora Sea. The place of observations is marked by red asterisk.

as effects of nonlinearity and dispersion. Because internal solitary waves are often of large amplitudes, it is sometimes useful to include a cubic nonlinear term in (1). A dimensional extension of Korteweg-de Vries model (1) that accounts for the cubic nonlinearity and Coriolis forcing due to Earth's rotation is the Gardner-Ostrovsky equation (also known as the extended rotation-modified KdV equation):

$$
\frac{\partial}{\partial x}\left(\frac{\partial \eta}{\partial t}+\left(c+\alpha \eta+\alpha_{1} \eta^{2}\right) \frac{\partial \eta}{\partial x}+\beta \frac{\partial^{3} \eta}{\partial x^{3}}\right)=\frac{f^{2}}{2 c} \eta,
$$

where $\eta(x, t)$ is an unknown function, $x$ is a spatial variable (horizontal coordinate), $t$ is time, $c$ is the relevant linear long wave speed, coefficients $\alpha, \alpha_{1}$, and $\beta$ of the nonlinear and dispersive terms, respectively, are the environmental parameters of internal waves determined by the properties of the basic state (all these coefficients are described below), and $f$ is the Coriolis parameter, which depends on the period of Earth's rotation $T_{e}=24 \mathrm{~h}$ and the geographical latitude $\varphi$ :

$$
f=\frac{4 \pi}{T_{e}} \sin \varphi .
$$

Corresponding numerical model of (3) is described in [15, 22]. This model was verified for the shelves of Australia and Europe [23] and was used for the study of internal solitary wave transformations on the shelves of the Kara Sea and the Laptev Sea; internal breathers were also modeled for the conditions of the Baltic Sea [24].

It is worthy to note that internal wave dynamics essentially depends on the signs of the coefficients of (3). The coefficient of dispersion $\beta$ is always positive; however, the coefficients of quadratic nonlinearity $\alpha$ and cubic nonlinearity $\alpha_{1}$ can have either sign and even may vanish. The possible combinations of the signs of the nonlinear coefficients in the Gardner equation correspond to different wave propagation regimes.

\section{Coefficients of the Gardner- Ostrovsky Model}

It was already mentioned that parameters of model (3) depend on the background density stratification (buoyancy frequency profile) and sea depth. As one can see from Figure 2, there are only a few points in vertical coordinate in 


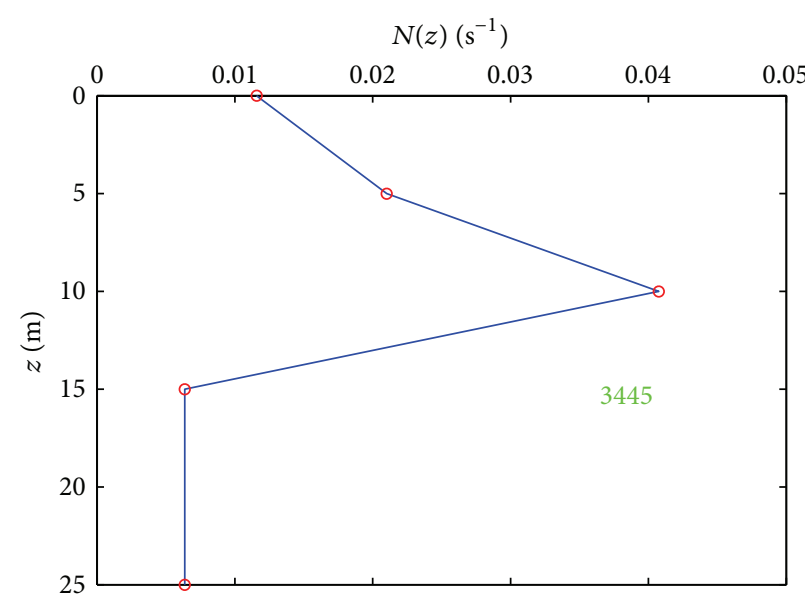

(a)

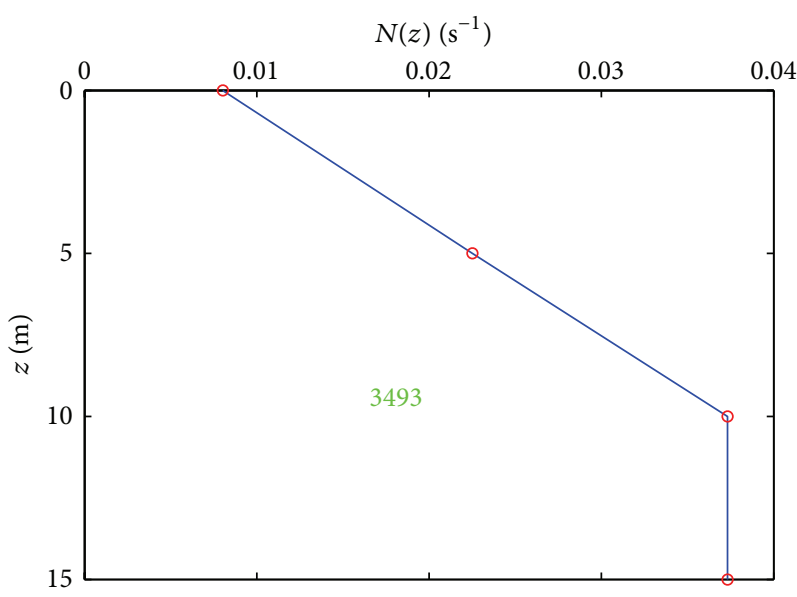

(b)

FIGURE 2: Buoyancy frequency profiles in the Pechora Sea near the region of observation of internal bore.

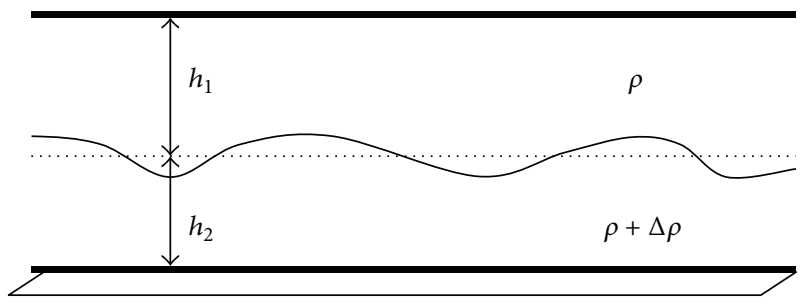

FIGURE 3: Scheme of the two-layer model.

the hydrological data, and this fact implies quite rough estimates of the parameters of (3). Therefore we (following original paper [16]) decided to use a two-layer approximation of vertical density profile (Figure 3 ).

For the case of two-layer stratification explicit expressions of the coefficients of the Gardner-Ostrovsky equation are known [25-27]:

$$
\begin{aligned}
c & =\sqrt{\frac{g \Delta \rho}{\rho} \frac{h_{1} h_{2}}{h_{1}+h_{2}}}, \\
\alpha & =\frac{3 c}{2} \frac{h_{1}-h_{2}}{h_{1} h_{2}}, \\
\beta & =\frac{c h_{1} h_{2}}{6}, \\
\alpha_{1} & =-\frac{3 c}{8 h_{1}^{2} h_{2}^{2}}\left(h_{1}^{2}+h_{2}^{2}+6 h_{1} h_{2}\right) .
\end{aligned}
$$

Here $h_{1}$ and $h_{2}$ are the undisturbed widths of the upper and lower layers, respectively, $\rho$ is the reference density, and $\Delta \rho$ is density jump between the layers. The sea depth is taken equal to $15 \mathrm{~m}$. The characteristics of the two-layer model are given in Table 1. The latitude to calculate the Coriolis parameter (4) was taken to be $69.5^{\circ} \mathrm{N}$. Coefficients (5) of (3) are given in Table $2(\Delta \rho / \rho=0.0027)$. They were calculated using the data from Table 1 .

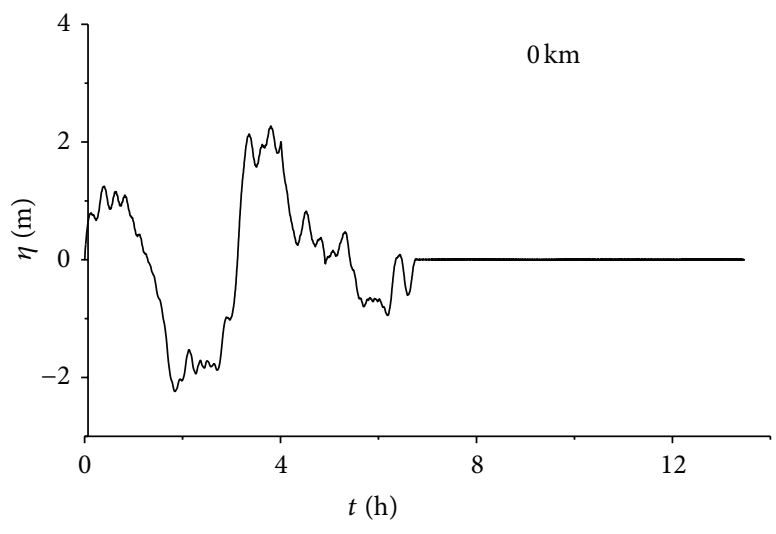

FIGURE 4: The shape of the initial condition (at $x=0 \mathrm{~km}$ ) for (3).

TABLE 1

\begin{tabular}{lccc}
\hline & Temperature & Salinity & Density \\
\hline $\begin{array}{l}\text { Upper layer, } \\
h_{1}=10 \mathrm{~m}\end{array}$ & $T_{1}=6^{\circ} \mathrm{C}$ & $S_{1}=30 \% 0$ & $\begin{array}{c}\rho_{1}\left(T_{1}, S_{1}\right)= \\
1023,60 \mathrm{~kg} / \mathrm{m}^{3}\end{array}$ \\
\hline $\begin{array}{l}\text { Lower layer, } \\
h_{2}=5 \mathrm{~m}\end{array}$ & $T_{2}=3^{\circ} \mathrm{C}$ & $S_{2}=33 \% 0$ & $\begin{array}{c}\rho_{2}\left(T_{2}, S_{2}\right)= \\
1026,36 \mathrm{~kg} / \mathrm{m}^{3}\end{array}$ \\
\hline
\end{tabular}

TABLE 2

\begin{tabular}{lc}
\hline Parameter & Value \\
\hline$c$ & $0.297 \mathrm{~m} / \mathrm{s}$ \\
$\beta$ & $2.475 \mathrm{~m}^{3} / \mathrm{s}$ \\
$\alpha$ & $0.0445 \mathrm{~s}^{-1}$ \\
$\alpha_{1}$ & $-0.0233(\mathrm{~m} \cdot \mathrm{s})^{-1}$ \\
\hline
\end{tabular}

Note that using of the two-layer approximation leads to an essential increase (up to two times and even more) of values of nonlinear and dispersive coefficients of (3) in comparison to similar continuous density stratification. The absolute value 

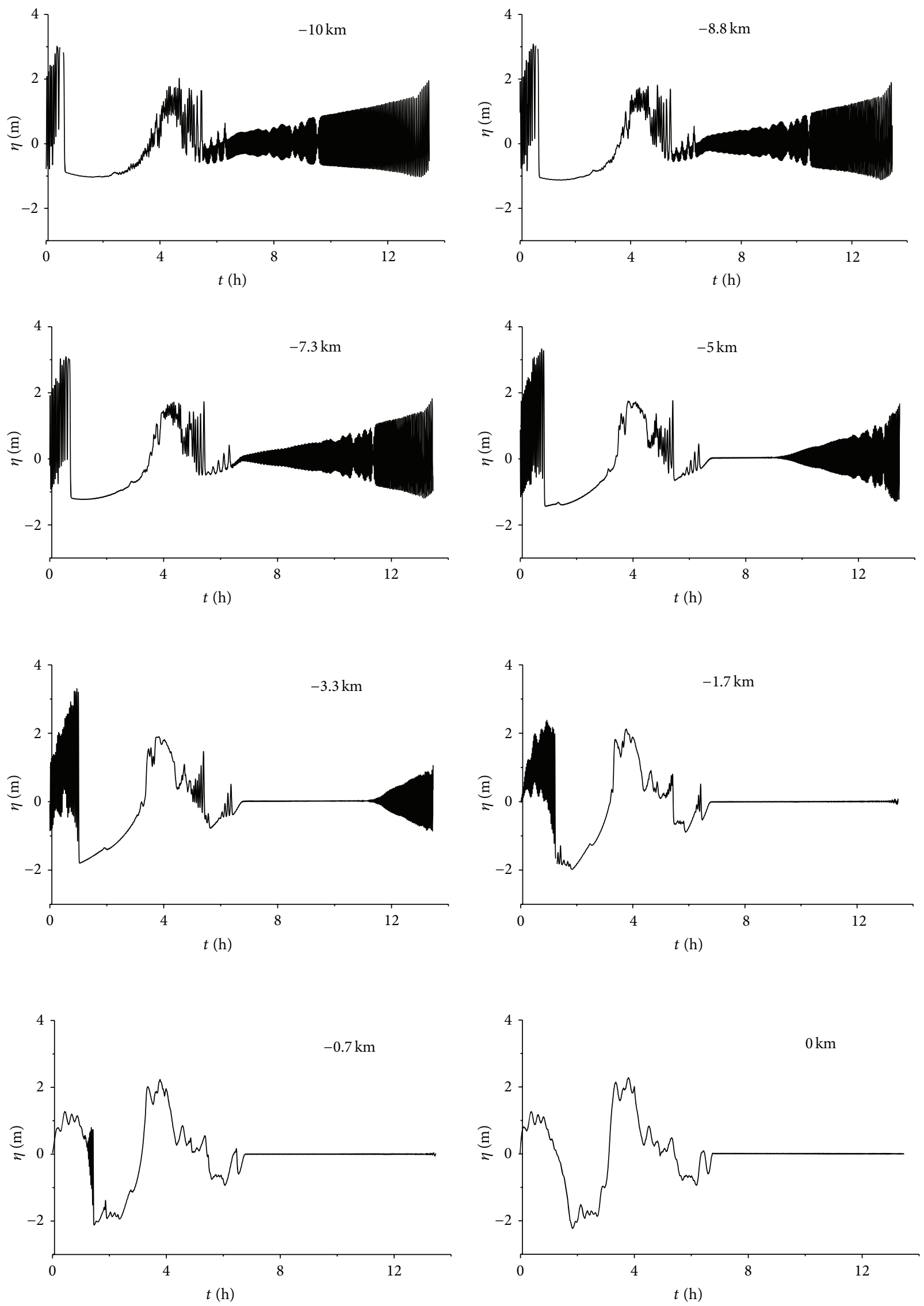

Figure 5: Continued. 

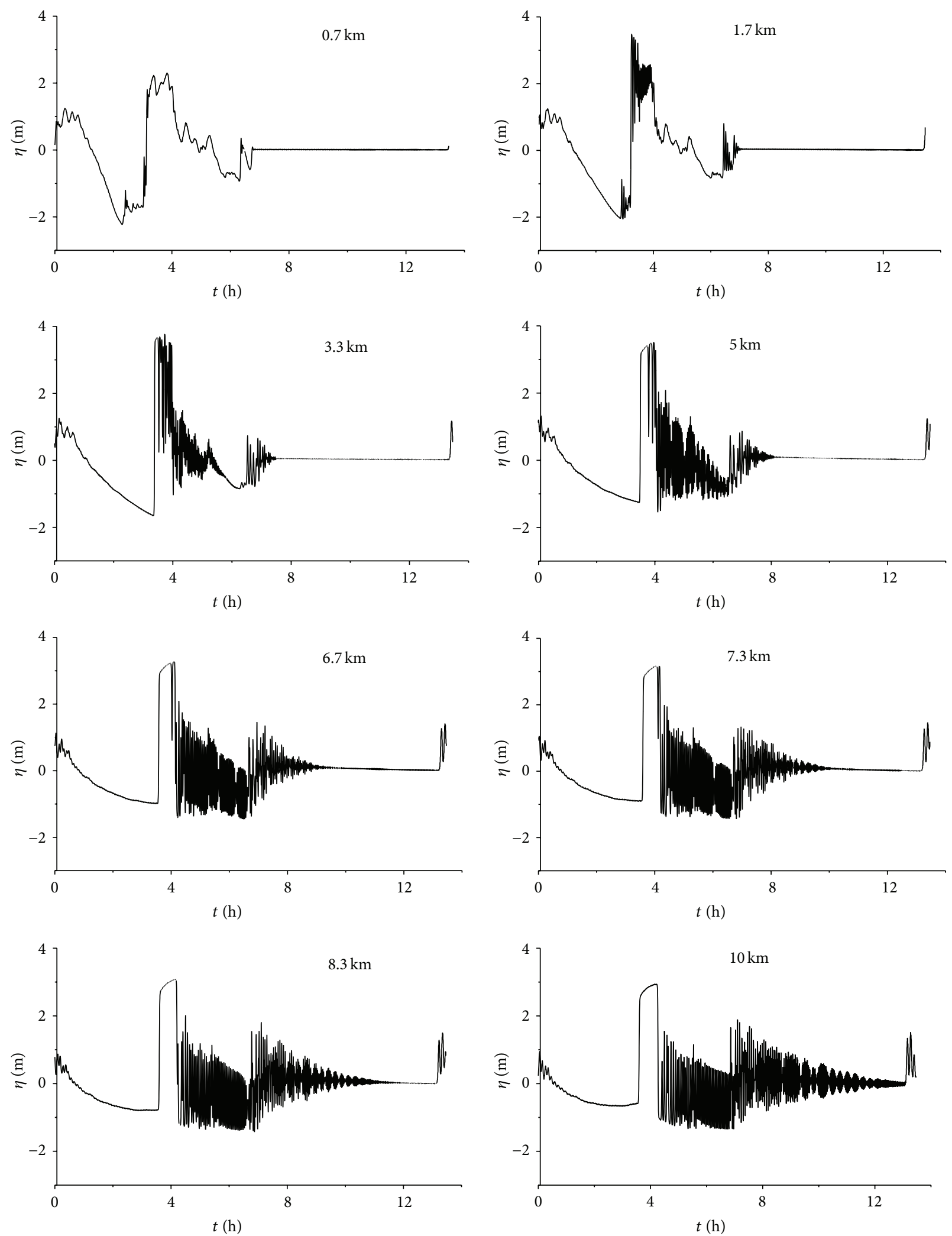

FIgURE 5: Transformation of internal wave from the point $x=-10 \mathrm{~km}$ to $x=10 \mathrm{~km}$. 
of coefficient $\alpha_{1}$ at the cubic nonlinear term here is comparable to the quadratic nonlinearity parameter $\alpha$. Therefore one can expect an increase of the influence of nonlinearity in such a model.

The internal wave record presented in [16] was digitized with the time step of 18 seconds. High-frequency nontidal oscillations and noise were filtered out with the help of standard algorithm given in [28]. The numerical model of the boundary problem for (3) was solved with periodical boundary conditions in time; therefore the time interval of the record was enlarged up to 13.5 hours. Also initial signal $\eta(x=$ $\left.x_{0}, t\right)=\eta_{0}(t)$ was recalculated from the given level of $10.54 \mathrm{~m}$ from the seabed to the level of the interface between layers in two-layer model ( $5 \mathrm{~m}$ from bottom). The shape of the initial condition is given in Figure 4.

\section{The Results of the Modeling}

The modeling of internal wave evolution was carried out with the aim of estimations of "lifetime" for certain forms of wave motion, in particular, for internal bore (not containing undulations) and solibore (with undulations). The initial wave was used either for calculations of positive $x$-direction or negative one (estimated prehistory, or past state of the wave). Transformation of the wave from the point $x=-10 \mathrm{~km}$ to $x=10 \mathrm{~km}$ is presented in Figure 5.

As the effect of cubic nonlinearity is significant for our model, it leads to generation of one thick (top-table) solitary wave (having maximal possibility for solitons amplitude) and a number of usual $\mathrm{KdV}$-solitary waves with amplitude being twice less than limiting one $\left(A_{\lim }=\left|\alpha / \alpha_{1}\right|[14]\right)$ in the process of degeneration of internal bore. This process was studied theoretically in [29]. The top-table solitary wave is clearly visible both at direct $(x=8.3 \mathrm{~km})$ and at inverse $(x=-10 \mathrm{~km})$ transformation of the initial bore-like wave. Thus, kink (steep wave front without undulations on its profile) is present at almost all the stages of the wave transformation. In fact, the wave shape, which is given in experimental record, is formed in the neighborhood of the point $x=-1.7 \mathrm{~km}$ and remains the same almost until the point $x=1.7 \mathrm{~km}$. It exists on the distance of about $3.5 \mathrm{~km}$ during around 3 hours.

The action of positive quadratic and significant negative cubic nonlinearity leads to asymmetry (to positive polarities) of the distribution function for wave displacements due to formation of solitons. In the vicinity of $x=0$, where there are no solitary waves, one can observe a deeper trough between two crests, while the amplitude of this trough decreases in the regions before and after $x=0$. This can be explained by positive quadratic and significant cubic nonlinearity when the formation of solitons leads to asymmetry of the distribution function of the displacement in the wave in the positive region.

\section{Conclusion}

Numerical calculations of the evolution of the field of internal waves in the Pechora Sea, in which measurements of internal bore were carried out in August 1998, are performed.
Measured form of internal bore is used as the initial condition for numerical simulations. Numerical model based on the Gardner equation allows enabling the forecast of wave evolution. It is shown that a sharp drop (kink) in the depth of the thermocline is saved at a distance of one-three kilometers, and then it is transformed into solibore (shock wave with undulations).

\section{Conflict of Interests}

The authors declare that there is no conflict of interests regarding the publication of this paper.

\section{Acknowledgment}

The presented results were obtained within the framework of the State Order in the sphere of scientific activity (Task $5.30 .2014 / \mathrm{K}$ ) and organization of scientific research (Task 2014/133).

\section{References}

[1] J. J. Stoker, Water Waves: The Mathematical Theory with Applications, Interscience Publishers, New York, NY, USA, 1957.

[2] G. B. Whitham, Linear and Nonlinear Waves, Wiley-Interscience, New York, NY, USA, 1974.

[3] C. M. Dafermos, Hyperbolic Conservation Laws in Continuum Physics, Springer, New York, NY, USA, 2009.

[4] T. B. Benjamin and M. J. Lighthill, "On conoidal waves and bores," Proceedings of the Royal Society-Series A: Mathematical, Physical and Engineering Sciences, vol. 224, pp. 448-460, 1954.

[5] M. J. Ablowitz, Nonlinear Dispersive Waves. Asymptotic Analysis and Solitons, Cambridge University Press, Cambridge, UK, 2011.

[6] A. V. Gurevich and L. P. Pitaevskii, "Nonstationary structure of a collisionless shock wave," Soviet Physics-JETP, vol. 38, pp. 291295, 1974.

[7] A. M. Kamchatnov, Nonlinear Periodic Waves and Their Modulations: An Introductory Course, World Scientific, Singapore, 2000.

[8] G. A. El, "Resolution of a shock in hyperbolic systems modified by weak dispersion," Chaos, vol. 15, no. 3, Article ID 037103, 2005.

[9] A. M. Kamchatnov, Y.-H. Kuo, T.-C. Lin et al., "Undular bore theory for the Gardner equation," Physical Review E-Statistical, Nonlinear, and Soft Matter Physics, vol. 86, no. 3, Article ID 036605, 2012.

[10] S. Myint and R. H. J. Grimshaw, “The modulation of nonlinear periodic wavetrains by dissipative terms in the Korteweg-de Vries equation," Wave Motion, vol. 22, no. 2, pp. 215-238, 1995.

[11] N. F. Smyth and P. E. Holloway, "Hydraulic jump and undular bore formation on a shelf break," Journal of Physical Oceanography, vol. 18, no. 7, pp. 947-962, 1988.

[12] V. Vlasenko, N. Stashchuk, and K. Hutter, Baroclinic Tides: Theoretical Modeling and Observational Evidence, Cambridge University Press, Cambridge, UK, 2005.

[13] J. R. Apel, L. A. Ostrovsky, Y. A. Stepanyants, and J. F. Lynch, "Internal solitons in the ocean and their effect on underwater sound," Journal of the Acoustical Society of America, vol. 121, no. 2, pp. 695-722, 2007. 
[14] R. Grimshaw, E. Pelinovsky, T. Talipova, and O. Kurkina, "Internal solitary waves: propagation, deformation and disintegration," Nonlinear Processes in Geophysics, vol. 17, no. 6, pp. 633-649, 2010.

[15] P. E. Holloway, E. Pelinovsky, and T. Talipova, "A generalized Korteweg-de Vries model of internal tide transformation in the coastal zone," Journal of Geophysical Research: Oceans, vol. 104, no. 8, pp. 18333-18350, 1999.

[16] G. I. Shapiro, V. P. Shevchenko, A. P. Lisitsyn, A. N. Serebryany, N. V. Politova, and T. M. Akivis, "Influence of internal waves on the suspended sediment distribution in the Pechora Sea," Doklady Earth Sciences, vol. 373, pp. 899-901, 2000.

[17] J. C. Sánchez-Garrido and V. Vlasenko, "Long-term evolution of strongly nonlinear internal solitary waves in a rotating channel," Nonlinear Processes in Geophysics, vol. 16, no. 5, pp. 587-598, 2009.

[18] K. R. Helfrich and W. K. Melville, "Long nonlinear internal waves," Annual Review of Fluid Mechanics, vol. 38, pp. 395-425, 2006.

[19] R. Grimshaw and K. Helfrich, "Long-time solutions of the Ostrovsky equation," Studies in Applied Mathematics, vol. 121, no. 1, pp. 71-88, 2008.

[20] Joint U.S.-Russian Atlas of the Arctic Ocean, University of Colorado, Boulder, Colo, USA, 1998.

[21] L. A. Ostrovsky, "Nonlinear internal waves in a rotating ocean," Oceanology, vol. 18, pp. 119-125, 1978.

[22] D. Y. Tyugin, O. E. Kurkina, and A. A. Kurkin, "Software package for the numerical simulation of the internal gravity waves in the ocean," Fundamental and Applied Hydrophysics, vol. 4, no. 2, pp. 32-44, 2011 (Russian).

[23] T. G. Talipova, E. N. Pelinovsky, A. A. Kurkin, and O. E. Kurkina, "Modeling of long internal wave dynamics on the shelf," Izvestiya, Atmospheric and Oceanic Physics, vol. 50, no. 6, pp. 714-722, 2014.

[24] E. Rouvinskaya, T. Talipova, O. Kurkina, T. Soomere, and D. Tyugin, "Transformation of internal breathers in the idealised shelf sea conditions," Continental Shelf Research. Submitted.

[25] V. D. Djordjevic and L. G. Redekopp, “The fission and disintegration of internal solitary waves moving over two-dimensional topography," Journal of Physical Oceanography, vol. 8, no. 6, pp. 1016-1024, 1978.

[26] T. Kakutani and N. Yamasaki, "Solitary waves on a two-layer fluid," Journal of the Physical Society of Japan, vol. 45, no. 2, pp. 674-679, 1978.

[27] C. G. Koop and G. Butler, "An investigation of internal solitary waves in a two-fluid system," Journal of Fluid Mechanics, vol. 112, pp. 225-251, 1981.

[28] V. Guerra and R. A. Tapia, "A local procedure for error detection and data smoothing," MRC Technical Summary Report 1452, Mathematics Research Center, University of Wisconsin, Madison, Wis, USA, 1974.

[29] R. Grimshaw, D. Pelinovsky, E. Pelinovsky, and A. Slunyaev, "Generation of large-amplitude solitons in the extended Korteweg-de Vries equation," Chaos, vol. 12, no. 4, pp. 1070-1076, 2002 . 

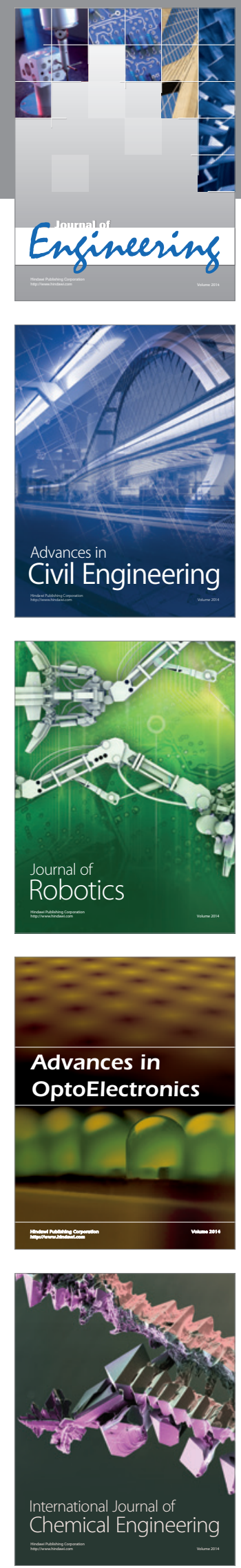

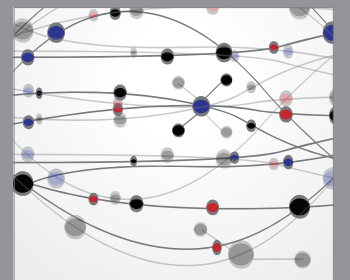

The Scientific World Journal
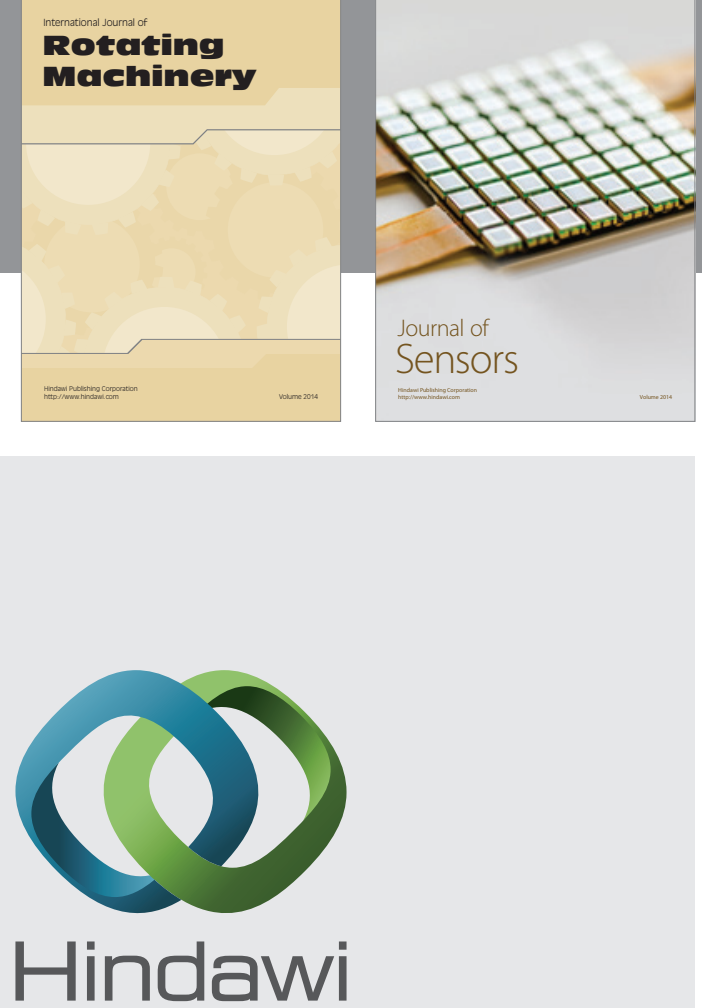

Submit your manuscripts at http://www.hindawi.com
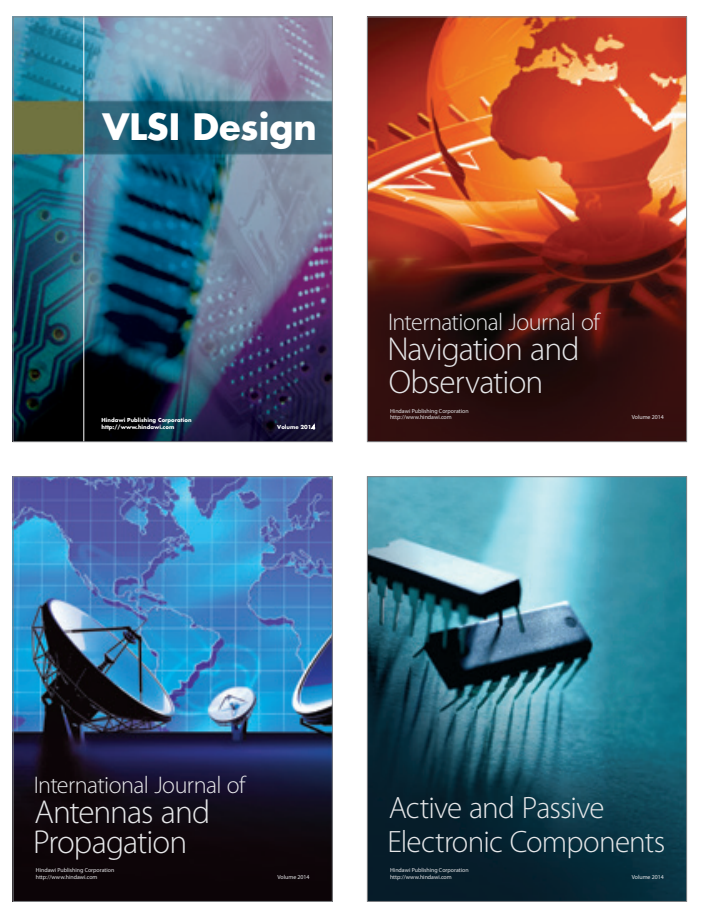
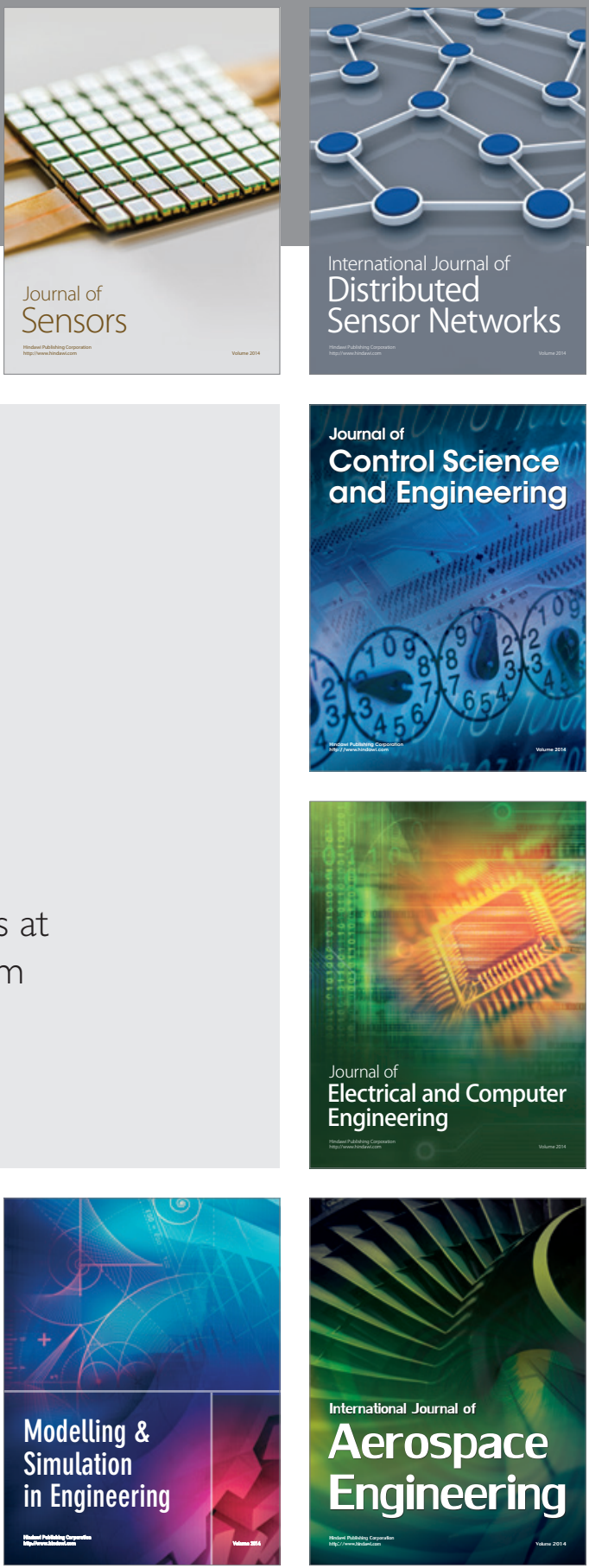

Journal of

Control Science

and Engineering
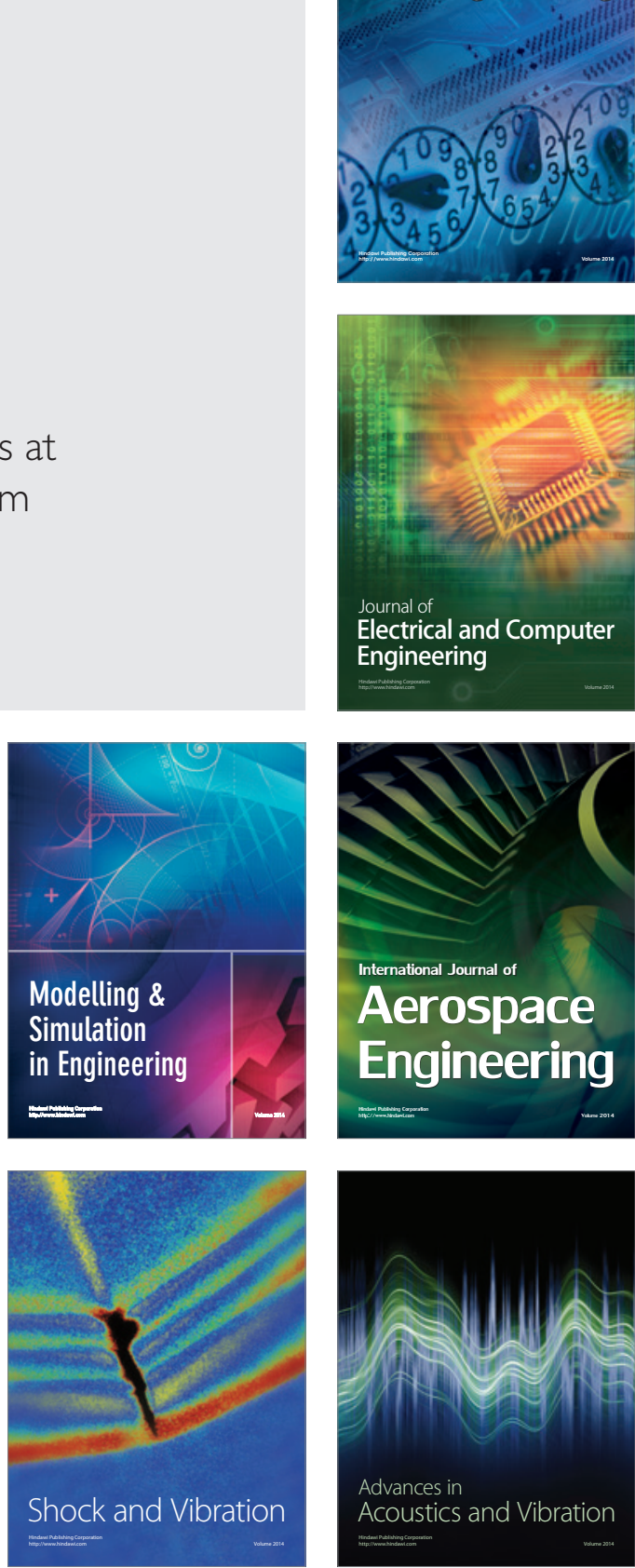